\title{
Semantic context effects in language production: $A$ swinging lexical network proposal and a review
}

\author{
Rasha Abdel Rahman \\ Humboldt-University Berlin, Germany \\ Alissa Melinger \\ University of Dundee, Scotland
}

\begin{abstract}
The investigation of semantic context effects has served as a valuable tool in investigating mechanisms of language production. Classic semantic interference effects have provided influential support for and interest in a competitive lexical selection mechanism. However, recent interest in semantic facilitation effects has stimulated a discussion on whether context effects reflect competition during lexical selection. In this review we propose a framework of lexical selection by competition that is sensitive to the activation of lexical cohorts. We outline our proposal and then present a selective review of the empirical evidence, much of which has been central to the development of alternative non-competitive models. We suggest that by adopting the assumptions of our proposal we can parsimoniously account for a majority of the discussed semantic facilitation and interference effects.
\end{abstract}

Investigations of semantic context effects, a widely used tool to study the architecture of the speech production system, have considerably shaped our assumptions on the nature of lexical selection. Traditionally the term semantic context has referred primarily to the relationship between a target stimulus and some second stimulus within an experiment. In this paper, we intend semantic context to refer to any meaning-constraining context, i.e., discourse context, experimental context, situational context, within which a target word is uttered.

Correspondence should be addressed to Rasha Abdel Rahman, Humboldt-University Berlin, Rudower Chaussee 18, 12489 Berlin, Germany. E-mail: rasha.abdel.rahman@cms.hu-berlin.de

Preparation of this paper was supported by a grant (AB 277 1-2) from the German Research Council to Rasha Abdel Rahman.

(C) 2009 Psychology Press, an imprint of the Taylor \& Francis Group, an Informa business http://www.psypress.com/lcp

DOI: $10.1080 / 01690960802597250$ 
Until recently there has been a strong focus in the literature on interference effects that are typically induced by categorical relations between stimuli. These well-attested effects have long been taken as evidence for competition among co-activated entries during lexical selection, an assumption that is shared by many production models (e.g., Bloem \& La Heij, 2003; Harley, 1993a, 1993b; Humphreys, Lloyd-Jones, \& Fias, 1995; La Heij, 1988; Levelt, Roelofs, \& Meyer, 1999; Roelofs, 1992; Starreveld \& La Heij, 1996). Recently, however, reported polarity reversals and exceptions from classic interference effects have gained increasing attention. Several pieces of evidence suggest that semantic interference is a rather narrow phenomenon, seemingly restricted to categorical relations. The absence of semantic interference effects or the presence of facilitation for various types of noncategorical relations is problematic for competitive models of lexical selection because they generally do not stipulate or restrict the spread of activation within the conceptual system or between conceptual and lexical strata to categorically related items (for an alternative view, see Bloem \& La Heij, 2003).

To resolve this contradiction, a recent proposal suggests that first, semantic interference does not reflect lexical selection mechanisms and that second, lexical selection is not by competition (Costa, Alario, \& Caramazza, 2005; Finkbeiner \& Caramazza, 2006; Janssen, Schirm, Mahon, \& Caramazza, 2008; Mahon, Costa, Peterson, Vargas, \& Caramazza, 2007; Miozzo \& Caramazza, 2003).

According to Mahon and colleagues' (2007) response exclusion hypothesis, semantic facilitation reflects lexical priming (but see Costa et al., 2005 for a proposal that assumes a conceptual locus) of the target whereas interference effects arise post-lexically, in the articulatory buffer, to which distractor words are presumed to have privileged access. The output buffer constitutes a bottleneck stage which, once occupied by a distractor word, must be disengaged before articulation of the target word can start. The ease with which the non-target response can be excluded from production depends on its response relevance. If the distractor is a relevant response in a given experimental context, for instance because it shares semantic category with the target, exclusion of the non-target response is slower. However, when the distractor is not response relevant (for instance, because it is semantically unrelated or because of implicit task criteria such as naming whole objects rather than parts of objects; cf. Costa et al., 2005), exclusion is easier and therefore faster. While this proposal is seemingly intended as a model of picture-word-interference performance, the authors also claim that the same mechanism can account for multiple interferencelike effects from a variety of paradigms (Mahon et al., 2007, p. 516).

The question of whether lexical selection is by competition is an important one for several reasons. As an explanatory mechanism, competition is broadly 
used across several different cognitive faculties. It plays a prominent role in various language functions, including word recognition (e.g., Gaskell \& Marslen-Wilson, 1997; McClelland \& Elman, 1986) sentence processing (e.g., Jurafsky, 1996; MacDonald, Pearlmutter, \& Seidenberg, 1994; McRae, Spivey, \& Tanenhaus, 1998), lexical ambiguity resolution (e.g., Duffy, Morris, \& Rayner, 1988; Rayner \& Duffy, 1986), Stroop conflicts (e.g., MacLeod, 1991), bilingual language processing (e.g., Green, 1986, 1998), as well as other components of the speech production process (e.g., syllable competition in the mental syllabary; Cholin, Levelt, \& Schiller, 2006; Roelofs, 1997). Furthermore, competition is not just a language-internal phenomenon; it is found as an explanatory device in various cognitive domains such as attentional processing (Duncan, 2001) and motor control (Georgopolous, Schwartz, \& Kettner, 1986; Houghton \& Tipper, 1994). Thus, determining whether competition is truly the mechanism underlying lexical selection in production has potential implications not just as a comprehensive explanation for a wide variety of experimental observations in production research but also for viewing the relationship between speech production and recognition, or other cognitive functions.

\section{A SWINGING LEXICAL NETWORK ACCOUNT OF SEMANTIC INTERFERENCE}

Here, we propose a theoretical account for semantic context effects that retains lexical competition. At the heart of this account are two assumptions that are either explicitly or implicitly incorporated in most current models of speech production. The first assumption is that the activation of an interrelated cohort of lexical competitors is a major determinant for lexical competition to be strong enough to result in detectable semantic interference effects. The activation of a single isolated competitor is typically not sufficient to induce measurable interference. This assumption is derived from models implementing selection mechanisms such as the Luce ratio (Levelt et al., 1999; Luce, 1959; Roelofs, 1992), however it may also be consistent with models that implement competition via inhibitory links between active representations (e.g., Caramazza, 1997; Harley, 1993a, 1993b; Howard, Nickels, Coltheart, \& Cole-Virtue, 2006; Stemberger, 1985). According to the Luce ratio, the probability for selecting the target lemma at any given point in time depends on the state of its activation relative to (divided by) the sum activation of all other lemma nodes (cf. Roelofs, 1992, 1997). Consequently, we assume that the latency of target lemma selection varies as a function of the state of activation of the entire lexical network, and is proportionally delayed with an increasing number of active competitors. 
The second assumption is that semantic context affects speech production at two processing levels. First, context affects conceptual processing due to semantic priming of the target concept. Second, semantic context influences lexical level processes due to competition between lexical entries for selection. These two effects yield a combination of facilitative and inhibitory influences (see Damian \& Als, 2005; Navarette \& Costa, 2005; Kuipers, La Heij, \& Costa, 2006 for similar assumptions). The polarity of observed net effects in naming latencies is the result of a trade-off between conceptual facilitation and lexical competition. Which of the two opposing effects wins the game strongly depends on whether or not an inter-related lexical cohort of sufficient size is activated. Because lexical entries need to be selected from among competitors whereas concepts simply receive more or less activation, we assume that an increasing number of co-activated concepts and lexical nodes has a stronger influence on lexical interference than on conceptual facilitation.

Semantic facilitation effects are widely interpreted as contextual and conceptual. For instance, context-induced facilitation effects motivated the message-congruency account (Kuipers et al., 2006; Kuipers \& La Heij, 2008) which holds that semantic facilitation is obtained when target and context converge on a single goal concept. In this account, convergence leads to conceptual facilitation, which can outweigh weaker lexical interference effects. Further support for the assumption that semantic contexts yield conceptual priming is found in a variety of studies revealing semantic facilitation effects (e.g., Bloem \& La Heij, 2003; Bloem, van den Boogaard, \& La Heij, 2004; Costa, Mahon, Savova, \& Caramazza, 2003; Glaser \& Düngelhoff, 1984; Kuipers \& La Heij, 2008; Kuipers et al., 2006). For example, Bloem and La Heij (2003) observed faster translation times for words (e.g., translating English $d o g$ into Dutch hond) superimposed on semantically related pictures (e.g., cat) compared with unrelated pictures (e.g., tree). They interpreted this facilitation effect as evidence that the distractor picture primed the semantic representation of the target word, speeding concept selection. Null effects - when interference was expected have also been taken as evidence for facilitation from a semantic context. Navarette and Costa (2005) failed to observe anticipated semantic interference effects in a picture-picture interference study and argued that the interference had been neutralised by a comparably sized priming effect at the conceptual level. While the swinging network model adopts a conceptual-level explanation for these semantic facilitation effects, other models attribute the effects to lexical level processes that are mediated by conceptual activation (cf. Mahon et al., 2007; Roelofs, 2006, 2008a, 2008b).

To summarise, we assume that semantic contexts always induce both conceptual facilitation and lexical competition. The net effects will be facilitation-dominant when only a single or small number of competitors is 
activated, in which case conceptual priming outweighs lexical competition. In contrast, effects will be interference-dominant when a large number of inter-related competitors, a lexical cohort, is active, in which case cohortinduced lexical competition outweighs conceptual priming. One related but novel augmentation is the proposal that semantic and lexical activation spread is highly dynamic, flexible and adjustable to the specific context in which the utterance is produced. As a result, the activation of lexical cohorts in the course of word production is strongly modulated by contextual factors. This contextual sensitivity is in line with notions of ad hoc category formation (Barsalou, 1983). Specifically, Barsalou proposed that novel categories are constructed to achieve goals. For example, when stranded on a desert island one will quickly construct an ad hoc category of 'things that catch rain water'. We suggest that the same mechanisms that underlie goal-driven ad hoc category formation drive the flexibility we observe within the conceptual and lexical system.

Although lexical cohort activation is theoretically not a new concept, the consequences for semantic context effects and lexical selection latencies have not gained much attention. Particularly, recent debates about the locus of semantic interference effects have failed to take this factor into account. Discussions have focused on the relative activation levels of the target and a single lexical competitor. However, while this concentration may have started for expository purposes, it has crept into the theorising that underlies some research.

However, as we will argue below, the lexical cohort account has the potential to explain a range of semantic context effects - including polarity reversals associated with different types of semantic relation - without requiring additional assumptions to restrict lexical competition, and without the need to dismiss lexical competition. For example, in the model proposed by Bloem and La Heij (2003; Bloem et al., 2004), only the target concept and target co-hyponyms spread activation to their corresponding lexical representations. This assumption is adopted to account for the absence of predicted interference effects. In contrast, in the present model semantic context effects of different polarity and strength follow naturally from the two assumptions described above and do not have to be attributed to qualitatively different underlying processes. Furthermore, this account makes some unique predictions about when interference effects from non-categorically related contexts should be observed.

In the following we present the predictions derived from a lexical cohort account and provide a selective review of evidence supporting these predictions. The major part of this review will focus on findings that have recently been interpreted as evidence against lexical competition models under the assumption that the activation status of a single competitor is the 
determining factor. We will re-evaluate this evidence in light of the cohort assumption.

\section{EVIDENCE FROM THE PICTURE-WORD INTERFERENCE (PWI) PARADIGM}

In the picture-word interference (PWI) paradigm a picture is presented for a naming response together with a to-be ignored word distractor. A classic and well-established finding in this paradigm is the semantic interference effect, that is, delayed naming latencies induced by categorically related, compared with unrelated words (e.g., Glaser \& Düngelhoff, 1984; Glaser \& Glaser, 1989; La Heij, 1988; Lupker, 1979; Lupker \& Katz, 1981, 1982; Schriefers, Meyer, \& Levelt, 1990).

Lexical competition models make two assumptions to account for this finding. First, in the course of picture naming, activation spreads at the conceptual level to the target and related concepts, which in turn activate their corresponding lexical nodes (e.g., Caramazza, 1997; Collins \& Loftus, 1975; Collins \& Quillian, 1969; Dell, 1986; Levelt et al., 1999; Roelofs, 1992, but see, e.g., Bloem \& La Heij, 2003). Second, lexical nodes compete with the target for selection. When a categorically related distractor is presented, its associated lexical node receives converging activation from the picture and the word, and will therefore, as a highly active competitor, delay target selection. As discussed above, this often described variant of lexical competition models focuses on the activation level of one strong competitor - the lexical entry of the distractor word - without directly taking the activation status of the lexical cohort into account.

Recent evidence for polarity reversals of semantic distractor effects has challenged the assumption of competitive lexical selection. Specifically, semantically related distractor words that are not members of the target's semantic category seem to induce facilitation rather than interference. For instance, several studies have demonstrated no effects or facilitation when target and distractor are associatively related (e.g., Alario, Segui, \& Ferrand, 2000; Bölte, Jorschick, \& Zwitserlood, 2003; La Heij, Dirkx, \& Kramer, 1990; Lupker, 1979). Facilitation has also been observed when targets and distractors have a part-whole relation (Costa et al., 2005).

Costa and colleagues (2005; see also Mahon et al., 2007) have interpreted such facilitation effects, including their own findings, as evidence against lexical competition because any semantic relation should induce interference, not just categorical relations. Instead, they argue that semantic contexts induce conceptual/lexical priming of the target, and that interference will only be observed when the distractors are potentially relevant responses that need to be discarded. If only whole objects are named in an experiment, then 
distractors that are parts of objects will be quickly dismissed as alternative responses. What remains is a semantic priming effect. A similar mechanism based on response relevance might hold for associative relations.

However, Abdel Rahman and Melinger (2007) have recently reported a study in which the associate distractors were also target pictures on other trials. They observed associatively induced facilitation despite the fact that the respective distractors were parts of the response set, and were therefore clearly relevant responses. Within one study, associatively related distractors induced facilitation whereas categorically related distractors induced classic interference effects. This finding cannot easily be explained purely in terms of the response relevant criteria. It would require the additional assumption that only categorical, but not associative, relations are processed and identified as relevant responses. Why this would be is not entirely clear.

Alternatively, the polarity reversals follow naturally from inherent differences between associative and categorical relations within the lexical cohort account. When target and distractor are members of the same category (e.g., bee and horse), they spread converging activation to a number of competitors, which share the category node and several semantic features (e.g., snake, mouse, fly, etc., cf. Figure 1). Thus, a whole cohort of interrelated competing lexical nodes is co-activated, and the network is swinging. With convergent activation, the interconnectivity of the cohort means that the bulk of the activation stays within the cohort, and each representation reinforces the activation of the whole set, resulting in comparatively high levels of activation, which in turn produce strong lexical competition effects.

In contrast, if target and competitor are associatively related but belong to different semantic categories (e.g., bee and honey), activation does not converge onto other related concepts because they do not share a common category node, and the number of other shared semantic features is low. Instead, activation from target and competitor concepts diverges onto mutually unrelated representations (e.g., bread, comb, and dessert). These activated concepts in turn pass their activation on to more and more unrelated concepts until the activation dissipates to insubstantial levels. This sort of divergent activation results in a wide-spread ripple of activation with two central loci of high activation at the origin, namely with target and distractor. This pattern of activation spreading gives rise to one-to-one competition within the lexical network, rather than one-to-many competition (cf. Figure 1).

As outlined above, the combined effects of conceptual priming and lexical competition will be facilitation-dominant when only one competitor is active (as is typically the case for associative relations), but interference-dominant when a lexical cohort is active (as is the case for categorical relations). 


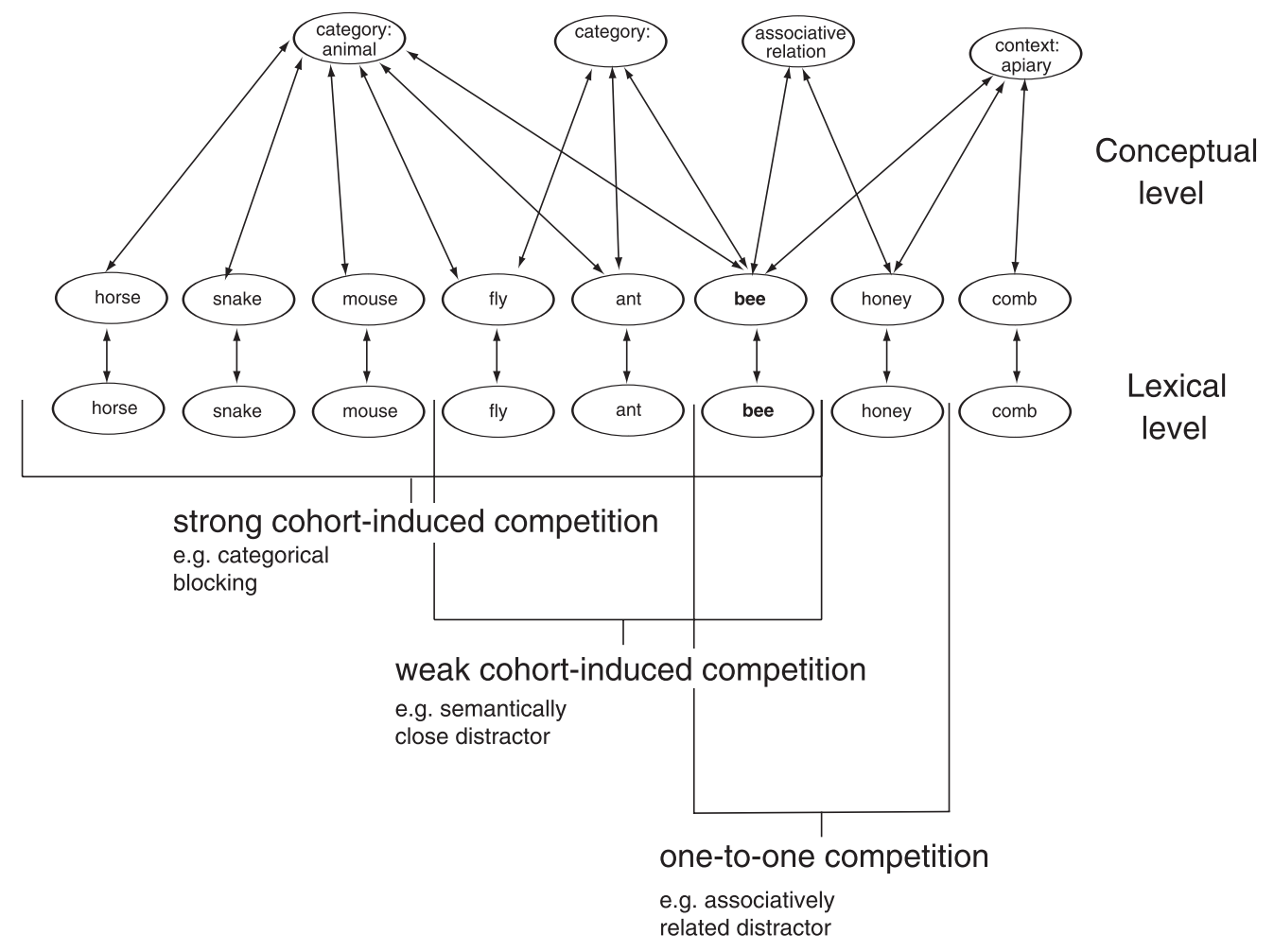

Figure 1. Schematic illustration of a cohort-based lexical competition model, adapted from Levelt et al. (1999). The fragment includes a conceptual and a lexical processing level. Arrows depict the bidirectional information transmission between the nodes of the network within and between the levels. For the sake of clarity, direct connections between conceptual nodes are not depicted. Depending on the semantic context, a target utterance (here: bee, in bold) can compete with a big or small lexical cohort or a single competitor.

Additionally, weak interference effects from a single competitor can also emerge when conceptual facilitation is absent (see below). The presentation of distractors that are parts of whole objects (e.g., car and bumper) might induce a one-to-one competition in a similar way as described for associates. This account for associative and categorical context effects receives further support from experiments with the semantic blocking paradigm (see below).

A further piece of evidence is that the presentation of semantically related verb distractors (e.g., target: bed, distractor: sleep) results in facilitation, rather than interference effects (Mahon et al., 2007). The authors have argued that this finding is at variance with lexical competition because such verb distractors are closely related to the target and should therefore receive strong converging activation, resulting in semantic interference, compared to unrelated verbs. However, in light of the cohort assumption again, this is not what would be expected. Just like associates, verb distractors such as 'sleep' and related targets (bed) systematically differ from categorically related noun distractors (chair) in that they tend to have a one-to-one semantic relation 
that will not yield significant converging activation within the lexical network. Consequently, conceptual priming will offset the one-to-one lexical competition.

Investigations of semantic distance effects are also relevant to the debate over lexical competition. The basic assumption here is again that the activation level of a single lexical competitor is the major determinant for semantic interference effects within lexical competition models. Based on this assumption, Mahon et al. (2007) have derived the prediction from lexical competition models that semantically close distractor words (e.g., target: horse, distractor: zebra) should interfere more with the naming response than semantically distant distractors (e.g., whale). However, what Mahon and colleagues observed was precisely the opposite: naming latencies associated with semantically distant distractors were slower than those associated with semantically close distractors. Other investigations of semantic distance have produced contrasting results, however. For example, Vigliocco, Vinson, Damian, and Levelt (2002a, 2002b) investigated effects of 'graded semantic similarity' using the semantic blocking paradigm. Because this paradigm differs from the PWI paradigm in important ways, particularly with respect to the activation of an inter-related lexical cohort, we will discuss this study in the following section on semantic blocking effects in more detail. We will include a discussion of the differences between the semantic distance effects observed by Mahon et al. and Vigliocco et al. in the section on blocking effects.

While the observation of slower naming latencies with increasing semantic distance might be viewed as being contrary to what a single-lexical competitor account would predict, it is in line with the above described trade-off account and competition induced by lexical cohorts. First, close distractors should yield stronger priming effects than distant distractors at the conceptual level. Second, one only needs to assume that semantically distant target-distractor pairs co-activate a lexical cohort with numerous different members belonging to this broadly defined category (e.g., animals: not only the target bee and distractor horse, but also other members of this category, such as ant, snake, mouse etc. are activated; cf. Figure 1). In contrast, semantically close target-distractor pairs (bee and fly) co-activate a much more confined and narrow category (e.g., insects: bee, fly, ant; cf. Figure 1). Such comparatively small semantic categories have fewer members than broad categories. Consequently, close distractors should induce less interference than distant distractors. While this claim is currently not supported by direct and independent empirical evidence, preliminary data point into this direction when the influences of semantic distance and category size effects are manipulated independently (Abdel Rahman \& Melinger, 2008b). 
The swinging network account proposed here incorporates the assumption that semantic activation spread can be modulated and adjusted in a situation-specific and flexible way. Support for this account, including some of the speculative aspects of the proposal, comes from studies that suggest a highly flexible architecture of the speech production system in terms of semantic activation spread. These studies show that meaning relations and contextually relevant categories can be formed, newly created, and even demolished by semantic context modulations (Abdel Rahman \& Melinger, 2007; Abdel Rahman \& Melinger, 2008b; see below). Thus, it is not unreasonable to assume that graded variations in target-distractor relations - such as semantic distance effects - strongly modulate the activation status of the whole lexical network. We will discuss this issue in more detail in the section on semantic blocking effects.

As mentioned above, the current proposal rests on two assumptions: activation of a cohort of lexical competitors and a trade-off between semantic facilitation and lexical interference effects. We suggest that a lexical cohort is necessary to produce sufficient competition to override conceptual facilitation effects; one-to-one competition will generally be insufficient to produce observable interference effects.

This proposal makes the prediction that even small interference effects from one-to-one competition should be observable if conceptual facilitation could be sufficiently reduced. Melinger and Abdel Rahman (2008) tested this hypothesis by investigating mediated semantic interference effects (Abdel Rahman \& Melinger, 2008a; Hantsch, Jescheniak, \& Schriefers, 2005; Jescheniak \& Schriefers, 1998; Levelt, Schriefers, Vorberg, Meyer, Pechmann, \& Havinga, 1991; Peterson \& Savoy, 1998). Such mediated effects are observed for distractor words that are phonologically related to a semantic competitor of the target. Melinger and Abdel Rahman used pairs of associatively related competitors in their study (e.g., target: pyramid, distractors: camera, bagel (phonologically related to the associate camel).

The authors reasoned that an interference effect should be detected because the phonological distractor avoids strong conceptual activation of the associate competitor, while increasing its lexical activation level. The lexical representation of the competitor receives activation from two sources: the target, due to spreading activation, and the distractor, via phonological priming. Crucially, however, the presentation of a pair of words that is phonologically related to an associatively related competitor (camera, bagel) should not significantly activate the conceptual representation of the competitor (camel). Thus, semantic facilitation is bypassed. As predicted by the lexical cohort hypothesis, in this situation, one-to-one lexical competition was sufficient to observe interference effects because these effects are not offset by conceptual facilitation. 
The present account is in line with similar effects for other types of relations such as near-synonyms (Jescheniak \& Schriefers, 1998) or category members (Abdel Rahman \& Melinger, 2008a). Because conceptual priming, semantic activation spread, and in turn lexical cohort activation is prevented by presenting distractors that are phonologically related to a competitor, a one-to-one lexical competition without concomitant conceptual facilitation should result in small but measurable interference effects.

Another common type of PWI polarity reversal that has received much attention is the so-called 'level of specificity' effect. In these studies, the target and distractor words describe objects at different levels of generality, i.e., animal (superordinate level), dog (basic level) or poodle (subordinate level). When the target and distractor differ in their respective levels of specificity, both facilitation (Costa et al., 2003; Glaser \& Düngelhoff, 1984; Kuipers et al., 2006; Vitkovitch \& Tyrrell, 1999) and interference (Hantsch et al., 2005; Glaser \& Düngelhoff, 1984; Kuipers et al., 2006) have been observed. Several different proposals have been put forward to explain the polarity of the effects. Some evidence suggests that the modality of the distractor presentation matters to the direction of the effect, with written distractors more likely to produce facilitation and auditory distractors more likely to produce interference (Hantsch, Jescheniak, \& Schriefers, 2006). It has also been argued that response congruency plays a role in determining the direction of these effects (Glaser \& Glaser, 1989; Kuipers et al., 2006; Kuipers \& La Heij, 2008; La Heij, Starreveld, \& Kuipers, 2007; Lupker \& Katz, 1981). While we do not believe that the entire pattern of results can be explained solely with reference to the current formulation of a swinging network and lexical cohort activation, we would like to speculate as to how the current approach could be extended to capture a large subset of the facilitation and interference effects.

A necessary assumption is that a semantic context, in this case the experimental naming instruction, can influence the dimensions along which activation resonates within the conceptual network. For example, when producing categorical labels such as 'animal' and 'furniture', semantic features that discriminate between members of each category should not be strongly activated. Rather, general features such as 'is a living thing', 'breathes', 'moves' will be active. Hence, the category node 'animal' will spread activation to other superordinate category nodes such as 'fish' and 'birds'. A basic level distractor, e.g., 'dog', will enhance the activation of the target 'animal' but it will not produce convergence because it is not related to the other categories (a dog is not a type of fish). As a result, the one-to-one competition at the lexical level will not be sufficient to overcome the semantic facilitation and the net result will be facilitative.

In contrast, interference effects are expected when the naming task is more specific than the distractor. In basic level naming, a target 'dog' will spread 
activation to competitors at the same level of specificity, e.g., 'cat', 'horse', 'tiger'. A superordinate distractor, e.g., 'animal', would be linked to many of the co-activated concepts, producing converging activation within the set of competitors, thus leading to enhanced cohort competition in the lexicon. Kuipers et al. (2006) reported exactly this predicted pattern of interference and facilitation effects. Furthermore, the same general approach explains several other level of specificity observations, but clearly not all of them (e.g., Costa et al., 2003, Experiments 1 and 2 but not 3; Glaser \& Glaser, 1989; Hantsch et al., 2005, Experiments 3 and 5 but not 1, 2 and 4; Vitkovitch \& Tyrrell, 1999, Experiments 1 and 3 but not 2).

\section{EVIDENCE FROM THE SEMANTIC BLOCKING PARADIGM}

In the semantic blocking paradigm pictures of objects are presented and named in blocks of trials containing either other members of a common semantic category (e.g., all objects are tools; homogeneous blocking condition) or unrelated objects from different categories (heterogeneous blocking condition; e.g., Belke, Meyer, \& Damian, 2005; Damian \& Als, 2005; Damian, Vigliocco, \& Levelt, 2001; Kroll \& Stewart, 1994; Vigliocco et al., 2002a, 2002b). When the objects are repeatedly presented, naming is slowed down in categorically homogeneous compared with heterogeneous blocks, paralleling the findings in the picture-word interference paradigm.

Just like the contextually induced interference effects in the PWI paradigm, blocking effects have long been thought to arise due to competition at the level of lexical selection. They are potentially also compatible with the non-lexical account described above, assuming that first, other objects in the homogeneous blocks are relevant responses - which they clearly are - and second, that 'previously named pictures will be available as potential responses' (Mahon et al., 2007, p. 516). Where precisely the potential alternative responses are available remains unclear. However, more difficult to explain along these lines is the observation of Damian and Als (2005), that semantic blocking effects are not affected by the presence of interspersed trials with unrelated objects or trials that contained nonlanguage tasks such as mental rotation. According to the output buffer assumption, this should reduce or even abolish interference effects because many previous responses are either unrelated, and thus not response relevant, or are not even associated with speech production. Yet, interference effects are unaffected. Damian and Als suggest an incremental learning mechanism for such long-lasting effects: Competitors - or more precisely the links between concepts and names - might be strengthened due to learning, and will thus subsequently produce more potent competitors. Such longlasting effects can be easily accommodated with our assumptions of dynamic 
network modulations. Therefore, and in line with Damian and Als (2005), we consider lexical competition as the most parsimonious explanation for semantic blocking effects.

Although the blocking paradigm can induce classic semantic interference effects, it differs from the PWI paradigm in important ways. Specifically, blocking allows for a flexible manipulation of semantic and lexical cohort activation. For instance, Belke and colleagues (2005) have demonstrated that semantic interference effects generalise to new, previously unnamed objects of the same semantic category. In line with the swinging network assumption, this finding suggests that blocking enhances the activation level of the whole semantic category, not just the activation of actually presented and named category members. Furthermore, the fact that the interference effects generalise to new category members supports the role of one-to-many competition over one-to-one competition.

Using the blocking paradigm, Abdel Rahman and Melinger (2007) have explored the specific mechanisms that underlie the polarity reversals observed for categorical and associative semantic relations. In these experiments, semantic interference was observed not only for homogeneous blocks consisting of category members (e.g., category: animals: bee, horse, mouse, etc.) but also for homogeneous blocks consisting of associates from different categories that belonged to a specific semantic context (e.g., context: apiary; bee, honey, comb, etc.), compared to heterogeneous blocks. Here, and in contrast to presenting an isolated distractor word, semantic blocking creates a situation in which related concepts and lexical entries synergistically activate each other. Thus, in the blocking paradigm lexical semantic interference effects are not restricted to category members but can also be found for associates from different categories because lexical cohorts can be activated for both types of relations. The observation that noncategorical relations can induce interference when a cohort of items is interrelated in a meaningful way supports the assumption that competition due to semantic contexts can be boosted by expanding one-to-one relations to oneto-many relations in a swinging lexical network.

Moreover, when the same material was presented in a classic picture-word interference situation, only categorically related distractors induced interference whereas associatively related distractors induced facilitation (see above for a more detailed description of these distractor effects). As argued, the reversal of semantic effects for associates in the PWI and semantic blocking paradigm, and the constant effects of categorical relations across paradigms, is a direct consequence of the specific nature of categorical and associative relations. Whereas category members more or less automatically induce converging activation of other category members (cf. Belke et al., 2005; Schriefers et al., 1990; but see below, Abdel Rahman \& Melinger, 
2008b), associates often have an isolated one-to-one relation, particularly when presented as target-distractor pairs in the PWI paradigm.

As discussed above, the competition induced by a single competitor, even when this competitor receives converging activation from the picture and itself, will not offset conceptual priming and will therefore not emerge as an interference effect. It is only when associatively related objects can be interrelated by providing a semantic context that the converging activation of a lexical cohort results in interference effects that are comparable with categorically induced effects. Together, these findings strongly support the lexical cohort assumption and suggest that semantic context effects induced by different types of semantic relations with diverging polarities are based on the same underlying mechanism: a trade-off between conceptual priming and lexical competition.

Another investigation of graded semantic similarity that is directly related to the above discussed graded interference effects reported by Mahon et al. (2007) comes from Vigliocco and colleagues (2002b). In a semantic blocking paradigm, participants named blocks of pictures drawn either from a single homogeneous category (e.g., body parts), two related categories (e.g., body parts and clothing) or two unrelated categories (e.g., body parts and vehicles). In contrast to Mahon et al's findings, objects in homogeneous blocks were named slower compared with objects in the block with two related categories, which in turn were named slower than objects in the block with two unrelated categories (but see Lotto, Job, \& Rumiati (1999) for potential effects of visual similarity on conceptual processing). Vigliocco et al.'s graded semantic effects also fall out from the assumptions of a highly flexible semantic network. Specifically, our proposal is that the semantic blocking paradigm mimics a constrained discourse context by increasing the relevance of links between contextually related concepts. The context can be thought of as an ad hoc category, e.g., 'things related to body parts', in which activation converges in a similar manner to taxonomic categories, thus inducing context effects that are not typically observed in paradigms such PWI. The result is an interference effect commensurate with the amount of lexical cohort activation.

Evidence that underscores the assumption that the spread of semantic activation during speech planning is highly flexible and adjustable to the semantic context comes from a recent blocking study (Abdel Rahman \& Melinger, 2008b). In this study, pictures of objects were presented either in categorically homogeneous blocks consisting of semantic category members (e.g., coffee, milk, rice, etc.), in thematically homogeneous blocks consisting of seemingly unrelated objects that could potentially be assigned to a common theme (e.g., 'fishing trip': coffee, knife, bucket, etc.), or heterogeneous blocks consisting of entirely unrelated objects (e.g., foods: coffee, shelf, bag, etc). 
As would be expected, a classic semantic interference effect for the categorically homogeneous condition was found, whereas naming latencies did not differ between the thematically homogeneous and the heterogeneous condition. However, when the blocks were preceded by visually presented matching title words that contextualised or inter-related the objects according to their category or theme, interference was observed for both types of homogeneous blocks. Thus, seemingly unrelated objects can induce interference if they are inter-related in a meaningful way. We assume that the relationship suggested by the block title facilitates ad hoc category formation (Barsalou, 1983). This ad hoc adaptation proceeds quickly and easily given a congruent title word. Furthermore, all interference effects, both from the categorical and thematic homogeneous blocks, were largely reduced when the homogeneous blocks were preceded by titles that were incongruent to the objects in the block. These observations indicate a high degree of plasticity and situation-specific adaptation of the composition of meaning-related concepts and lexical cohorts. This demonstration of a flexible adjustment of semantic spread of activation provides supporting evidence for our claim that ad hoc adaptations can strongly modulate the strength and polarity of semantic context effects by recruiting different lexical cohort assemblies of varying size.

\section{DISCUSSION AND CONCLUSIONS}

In this paper we proposed a swinging lexical network account for semantic context effects in speech production and provided a selective review on experimental evidence supporting this account. The proposal combines two assumptions. First, the specific characteristics of semantic activation spread during language production are dynamically modulated by semantic context. The empirical evidence reviewed here suggests a high degree of plasticity in that situation-specific adjustments to conceptual and lexical network activation unfold: semantic contexts modulate the speed of lexical selection by flexibly recruiting context-specific lexical cohort assemblies of varying sizes (Abdel Rahman \& Melinger, 2007; 2008b; Belke et al., 2005; Spalek \& Damian, 2007).

The second assumption is that the strength and polarity of semantic context effects depend on the outcome of a trade-off between semantically induced conceptual priming and lexical competition. Most importantly, the crucial factor that determines whether facilitation or interference effects dominate is the activation of an inter-related lexical cohort that strongly competes with the target for selection. Only when such a context-sensitive cohort is active, that is, when the lexical network is swinging, will lexical competition outweigh conceptual priming - and semantic interference effects 
be observed. We have reviewed several pieces of evidence from the PWI and semantic blocking paradigms suggesting that the activation status of the entire lexical network is a major determinant for interference effects induced by various types of semantic relations (e.g., Abdel Rahman \& Melinger, 2007; 2008b; Belke et al., 2005; Damian \& Als, 2005; Melinger \& Abdel Rahman, 2008). The circumstances where we predict interference to dominate facilitation are the following: (a) when a cohort of lexical candidates is active and/or (b) when conceptual facilitation is absent or strongly reduced. When neither of these criteria is satisfied, we expect facilitation. Here, as a basis for further empirical tests, we have discussed initial evidence supporting the above predictions. What further remains to be determined are the precise computational dynamics controlling the trade-off between interference and facilitation. For example, we do not know how large a lexical cohort must be before it can offset a conceptual facilitation effect. We also do not understand the full implications of assuming a conceptual, as opposed to a lexical, locus for semantic facilitation. It remains to be seen whether a lexical locus mediated by conceptual activation (as assumed by Roelofs, 2006, 2008a, 2008b) would also be consistent with the observed effects. Further specification will require computational modelling.

As discussed, the swinging network proposal is not an entirely new concept. Lexical cohort activation is an ingredient of many existing speech production models (most explicitly in Levelt et al., 1999; Roelofs, 1992, 1993, 2001, 2003, but also implicit in, e.g., Caramazza, 1997; Bloem \& La Heij, 2003; Humphreys et al., 1995; Starreveld \& La Heij, 1995, 1996). However, the impact of one-to-many competition induced by semantic contexts has not received much attention. The swinging network proposal does not require entirely new or qualitatively different assumptions for the effects of different types of semantic relations, (e.g., the assumption that the flow of activation between conceptual and lexical levels is restricted; Bloem \& La Heij, 2003; Bloem et al., 2004; Kuipers et al., 2006; Kuipers \& La Heij, 2008), and yet it covers a surprising range of data on semantic context effects, including many of the polarity reversals and exceptions from classic interference effects that have been interpreted as contra lexical competition models (e.g., Costa et al., 2005; Mahon et al., 2007). The gains in explanatory potential afforded by the swinging network proposal are achieved without the need to dismiss lexical competition, and without the need to attribute the underlying mechanisms to qualitatively different processes. Furthermore, although many of the reviewed context effects were facilitative, not inhibitory, the swinging network assumption can accommodate these observations while retaining lexical competition as an inherent element of lexical selection.

The review provided here is admittedly selective and clearly not exhaustive. It focuses strongly on recent evidence that has challenged lexical 
competition. The aim was to reinterpret this evidence in light of the cohort assumption, and in a broader sense to evaluate the scope of the assumption. Clearly, cohort competition cannot account for all reported polarity reversals. Specifically, it cannot explain recently reported reversed effects of masked distractor words (Finkbeiner \& Caramazza, 2006) or the effects of distractor words on delayed naming (Janssen et al., 2008). Furthermore, alternative proposals, such as the role of response congruency between target and distractor (Glaser \& Glaser, 1989; Kuipers et al., 2006; La Heij et al., 2007; Lupker \& Katz, 1981), or differences in distractor modality (Hantsch et al., 2006) might play as much of a role in determining the direction of distractor effects at different levels of specificity as our speculative extension of the swinging network account does.

While some of the reviewed studies were directly designed to test for cohort activation, many observations were not. In the latter case, we provided speculations on how the data can be interpreted within a swinging network account. Although this approach has yielded an interesting new perspective on the microstructure of speech planning and how this microstructure is modulated by semantic context, more direct support, including independent empirical tests and computational simulations of the network structure, would facilitate further evaluation of the proposal. To this end, we can identify several unique predictions derived from the swinging lexical network proposal. Some of these predictions received support from the studies describe above while others are yet untested. These predictions should contribute to the future development of this research.

First, all types of semantic contexts (e.g., categorical relations, associative relations, and even unrelated items that are linked in a meaningful way) can in principle induce interference as well as facilitation effects. The polarity depends on whether conceptual facilitation or lexical cohort activation prevails. Thus, factors that enhance lexical cohort activation should cause interference. Factors that induce semantic priming but fail to induce cohort activation should cause facilitation. Factors that are designed to induce even strong activation of one isolated competitor while still priming the target concept should produce facilitation or null effects.

Further predictions pertain to speech errors, such as naturally occurring non-categorical semantic substitutions, or semantic paraphasia. We have argued that a semantic context can induce a cohort effect for various types of semantic relations. In natural discourse, constrained topics should suffice to create converging activation akin to what is observed in a blocked picture naming experiment. Specifically, in a constrained conversation about farms, concepts within that semantic field, e.g., barn, plow, field, etc. will be salient and convergent. Hence, we would expect semantic substitutions between these contextually related items, e.g., barn replaced by plow. In contrast, in an unconstrained conversation semantic substitutions of contextually related 
words would not be expected. This prediction has yet to be directly tested, although naturally occurring associatively related semantic substitution errors have been reported (Harley \& MacAndrews, 2001). More detailed analyses of naturally occurring semantic substitution errors could be a valuable avenue for revealing additional evidence for the swinging network and lexical cohort activation.

We have presented a new framework for explaining both facilitative and inhibitory semantic effects in picture naming. Our proposal builds on assumptions that are implicit, but thus far largely neglected, in existing models. First, we reemphasised the importance of lexical cohorts to the competition mechanism underlying lexical selection. Second, we recognised that the semantic context affects word production both at the conceptual level, where priming effects arise, and at the lexical level, where interference arises. We also outlined the flexible manner with which the conceptual network can recruit various context-appropriate concepts into a lexical cohort; lexical cohorts can comprise concepts linked by common categories, contexts, and themes so long as the activation within the system is sufficiently convergent. We suggest that this notion of a flexible swinging network is crucial to an understanding of semantic context effects observed across a variety of experimental paradigms. In a selective review of recent key findings on semantic context effects we argued that the framework provides significant explanatory coverage, even of results previously thought to be incompatible with selection by competition, and makes unique testable predictions.

\section{REFERENCES}

Abdel Rahman, R., \& Melinger, A. (2007). When bees hamper the production of honey: Lexical interference from associates in speech production. Journal of Experimental Psychology: Learning, Memory and Cognition, 33, 604-614.

Abdel Rahman, R., \& Melinger, A. (2008a). Enhanced phonological facilitation and traces of concurrent word form activation in speech production: An object naming study with multiple distractors. Quarterly Journal of Experimental Psychology, 61, 1410-1440.

Abdel Rahman, R., \& Melinger, A. (2008b). The dynamic microstructure of speech production: Semantic interference built on the fly. Manuscript submitted for publication.

Alario, F.-X., Segui, J., \& Ferrand, L. (2000). Semantic and associative priming in picture naming. Quarterly Journal of Experimental Psychology, 53A, 741-764.

Barsalou, L. W. (1983). Ad hoc categories. Memory and Cognition, 11, 211-227.

Belke, E., Meyer, A. S., \& Damian, M. F. (2005). Refractory effects in picture naming as assessed in a semantic blocking paradigm. Quarterly Journal of Experimental Psychology, 58 A, 667-692.

Bloem, I., \& La Heij, W. (2003). Semantic facilitation and semantic interference in word translation: Implications for models of lexical access in language production. Journal of Memory and Language, 48, 468-488. 
Bloem, I., van den Boogaard, S., \& La Heij, W. (2004). Semantic facilitation and semantic interference in language production: Further evidence for the conceptual selection model of lexical access. Journal of Memory and Language, 51, 307-323.

Bölte, J., Jorschick, A., \& Zwitserlood, P. (2003). Reading yellow speeds up naming a picture of a banana: Facilitation and inhibition in picture-word interference. Proceedings of the European Cognitive Science Conference, Germany (pp. 55-60). Mahwah, NJ: Lawrence Erlbaum Associates Inc.

Caramazza, A. (1997). How many levels of processing are there in lexical access? Cognitive Neuropsychology, 14, 177-208.

Cholin, J., Levelt, W. J. M., \& Schiller, N. O. (2006). Effects of syllable frequency in speech production. Cognition, 99, 205-235.

Collins, A. M., \& Loftus, E. (1975). A spreading-activation theory of semantic processing. Psychological Review, 82, 407-428.

Collins, A. M., \& Quillian, M. R. (1969). Retrieval time from semantic memory. Journal of Verbal Learning and Verbal Behaviour, 8, 240-247.

Costa, A., Alario, F.-X., \& Caramazza, A. (2005). On the categorical nature of the semantic interference effect in the picture-word interference paradigm. Psychonomic Bulletin and Review, $12,125-131$.

Costa, A., Mahon, B., Savova, V., \& Caramazza, A. (2003). Level of categorization effect: a novel effect in the picture-word interference paradigm. Language and Cognitive Processes, 18, 205233.

Damian, M. F., \& Als, L. C. (2005). Long-lasting semantic context effects in the spoken production of object names. Journal of Experimental Psychology: Learning, Memory, and Cognition, 31, 1372-1384.

Damian, M. F., Vigliocco, G., \& Levelt, W. J. M. (2001). Effects of semantic context in the naming of pictures and words. Cognition, 81, B77-B86.

Dell, G. S. (1986). A spreading-activation theory of retrieval in sentence production. Psychological Review, 3, 283-321.

Duffy, S. A., Morris, R. K., \& Rayner, K. (1988). Lexical ambiguity and fixation times in reading. Journal of Memory and Language, 27, 429-446.

Duncan, J. (2001). An adaptive coding model of neural function in prefrontal cortex. Nature Reviews Neuroscience, 2, 820-829.

Finkbeiner, M., \& Caramazza, A. (2006). Now you see it, now you don't: On turning semantic interference into facilitation in a Stoop-like task. Cortex, 6, 790-796.

Gaskell, M. G., \& Marslen-Wilson, W. D. (1997). Integrating form and meaning: A distributed model of speech perception. Language and Cognitive Processes, 12, 613-656.

Georgopoulos, A. P., Schwartz, A. B., \& Kettner, R. E. (1986). Neuronal population coding of movement direction. Science, 233, 1416-1419.

Glaser, W. R., \& Düngelhoff, F.-J. (1984). The time course of picture-word interference. Journal of Experimental Psychology: Human Perception and Performance, 10, 640-654.

Glaser, W. R., \& Glaser, M. O. (1989). Context effects on Stroop-like word and picture processing. Journal of Experimental Psychology: General, 118, 13-42.

Green, D. W. (1986). Control, activation and resource. Brain and Language, 27, 210-223.

Green, D. W. (1998). Mental control of the bilingual lexico-semantic system. Bilingualism: Language and Cognition, 1, 67-81.

Hantsch, A., Jescheniak, J. D., \& Schriefers, H. (2005). Semantic competition between hierarchically related words during speech planning. Memory and Cognition, 33, 984-1000.

Hantsch, A., Jescheniak, J. D., Schriefers, H., \& Aßmann, S. (2006). Effects of distractor modality on semantic between-level effects in the picture-word-interference paradigm. Abstracts of the 47th Annual Meeting of the Psychonomic Society, USA, 11, 71. 
Hantsch, A., Jescheniak, J. D., \& Schriefers, H. (2006, August). Semantic facilitation in the pictureword-interference paradigm: Implications for models of lexical access. Paper presented at the International Workshop on Language Production, Chicago, IL, USA.

Harley, T. A. (1993a). Connectionist approaches to language disorders. Aphasiology, 7, 221-249.

Harley, T. A. (1993b). Phonological activation of semantic competitors during lexical access in speech production. Language and Cognitive Processes, 8, 291-309.

Harley, T. A., \& McAndrews, S. B. G. (2001). Constraints upon word substitution speech errors. Journal of Psycholinguistic Research, 30, 395-418.

Houghton, G., \& Tipper, S. P. (1994). A model of inhibitory mechanisms in selective attention. In D. Dagenbach \& T. Carr (Eds.), Inhibitory mechanisms in attention, memory and language (pp. 53-112). San Diego, CA: Academic Press.

Howard, D., Nickels, L., Coltheart, M., \& Cole-Virtue, J. (2006). Cumulative semantic inhibition in picture naming: Experimental and computational studies. Cognition, 100, 464-482.

Humphreys., G. W., Lloyd-Jones, T. J., \& Fias, W. (1995). Semantic interference effects on naming using a postcue procedure: Tapping the links between semantics and phonology with pictures and words. Journal of Experimental Psychology: Learning, Memory, and Cognition, 21, 961-980.

Janssen, N., Schirm, W., Mahon, B. Z., \& Caramazza, A. (2008). Semantic interference in a delayed naming task: Evidence for the response exclusion hypothesis. Journal of Experimental Psychology: Learning, Memory, and Cognition, 34, 249-256.

Jurafsky, D. (1996). A probabilistic model of lexical and syntactic access and disambiguation. Cognitive Science, 20, 137-194.

Kroll, J. F., \& Stewart, E. (1994). Category interference in translation and picture naming: Evidence for asymmetric connections between bilingual memory representations. Journal of Memory and Language, 33, 149-174.

Kuipers, J.-R., \& La Heij, W. (2008). Semantic facilitation in category and action naming: Testing the message-congruency account. Journal of Memory and Language, 58, 123-139.

Kuipers, J.-R., La Heij, W., \& Costa, A. (2006). A further look at semantic context effects in language production: The role of response congruency. Language and Cognitive Processes, 21, 892-919.

Jescheniak, J. D., \& Schriefers, H. (1998). Serial discrete versus cascaded processing in lexical access in speech production: Further evidence from the co-activation of near-synonyms. Journal of Experimental Psychology: Learning, Memory, and Cognition, 24, 1256-1274.

La Heij, W. (1988). Components of Stroop-like interference in picture naming. Memory and Cognition, 16, 400-410.

La Heij, W., Dirkx, J., \& Kramer, P. (1990). Categorical interference and associative priming in picture naming. British Journal of Psychology, 81, 511-525.

La Heij, W., Starreveld, P. A., \& Kuipers, J.-P. (2007). Structural complexity is not the (big) issue: A reply to Roelofs (2007). Language and Cognitive Processes, 22, 1261-1280.

Levelt, W. J. M., Roelofs, A., \& Meyer, A. S. (1999). A theory of lexical access in speech production. Brain and Behavioral Sciences, 22, 1-38.

Levelt, W. J. M., Schriefers, H., Vorberg, D., Meyer, A. S., Pechmann, T., \& Havinga, J. (1991). The time course of lexical access in speech production: A study of picture naming. Psychological Review, 98, 122-142.

Lotto, L., Job, R., \& Rumiati, R. (1999). Visual effects in picture and word categorization. Memory and Cognition, 27, 674-684.

Luce, R. D. (1959). Individual Choice Behavior. New York: John Wiley \& Sons.

Lupker, S. J. (1979). The semantic nature of response competition in the picture-word interference task. Memory and Cognition, 7, 485-495.

Lupker, S. J., \& Katz, A. N. (1981). Input, decision, and response factors in picture-word interference. Journal of Experimental Psychology: Human Learning and Memory, 7, 269-282. 
Lupker, S. J., \& Katz, A. N. (1982). Can automatic picture processing influence word judgments? Journal of Experimental Psychology: Human Learning and Memory, 8, 418-434.

Mahon, B. Z., Costa, A., Peterson, R., Vargas, K. A., \& Caramazza, A. (2007). Lexical selection is not by competition: A reinterpretation of semantic interference and facilitation effects in the picture-word interference paradigm. Journal of Experimental Psychology: Learning, Memory and Cognition, 33, 503-535.

McClelland, J. L., \& Elman, J. L. (1986). The TRACE model of speech perception. Cognitive Psychology, 18, 1-86.

MacDonald, M. C., Pearlmutter, N. J., \& Seidenberg, M. S. (1994). Lexical nature of syntactic ambiguity resolution. Psychological Review, 101, 676-703.

MacLeod, C. M. (1991). Half a century of research on the Stroop effect: An integrative approach. Psychological Bulletin, 109, 163-203.

McRae, K., Spivey-Knowlton, M. J., \& Tanenhaus, M. K. (1998). Modeling the influence of thematic fit (and other constraints) in on-line sentence comprehension. Journal of Memory and Language, 38, 283-312.

Melinger, A., \& Abdel Rahman, R. (2008). Lexical competition induced by indirectly activated semantic associates during picture naming. Manuscript submitted for publication.

Miozzo, M., \& Caramazza, A. (2003). When more is less - a counterintuitive effect of distractor frequency in picture-word interference paradigm. Journal of Experimental Psychology: General, $132,228-252$.

Navarrete, E., \& Costa, A. (2005). Phonological activation of ignored pictures: Further evidence for a cascade model of lexical access. Journal of Memory and Language, 53, 359-377.

Peterson, R. R., \& Savoy, P. (1998). Lexical selection and phonological encoding during language production: Evidence for cascaded processing. Journal of Experimental Psychology: Learning, Memory, and Cognition, 24, 539-557.

Rayner, K., \& Duffy, S. (1986). Lexical complexity and fixation times in reading: Effects of word frequency, verb complexity, and lexical ambiguity. Memory and Cognition, 14, 191-201.

Roelofs, A. (1992). A spreading-activation theory of lemma retrieval in speaking. Cognition, 42, $107-142$.

Roelofs, A. (1993). A spreading-activation theory of lemma retrieval in speaking. In W. J. M. Levelt (Ed.), Lexical access in speech productiom (pp. 107-142). Cambridge, MA: Blackwell.

Roelofs, A. (1997). The WEAVER model of word-form encoding in speech production. Cognition, 64, 249-284.

Roelofs, A. (2001). Set size and repetition matter: comment on Caramazza and Costa (2000). Cognition, 80, 283-290.

Roelofs, A. (2003). Goal-referenced selection of verbal action: Modeling attentional control in the Stroop task. Psychological Review, 110, 88-125.

Roelofs, A. (2006). Context effects of pictures and words in naming objects, reading words, and generating simple phrases. Quarterly Journal of Experimental Psychology, 59A, 1764-1784.

Roelofs, A. (2008a). Tracing attention and the activation flow in spoken word planning using eye movements. Journal of Experimental Psychology: Learning, Memory, and Cognition, 34, 353368.

Roelofs, A. (2008b). Dynamics of the attentional control of word retrieval: Analyses of response time distributions. Journal of Experimental Psychology: General, 137, 303-323.

Schriefers, H., Meyer, A. S., \& Levelt, W. J. M. (1990). Exploring the time course of lexical access in production: Picture-word interference studies. Journal of Memory and Language, 29, 86-102.

Spalek, K., \& Damian, M. F. (2007, August). Limited attentional resources affect semantic facilitation effects in object naming. Poster presented at the International Workshop on Language Production, Münster, Germany. 
Starreveld, P. A., \& La Heij, W. (1995). Semantic interference, orthographic facilitation, and their interaction in naming tasks. Journal of Experimental Psychology: Learning, Memory, and Cognition, 21, 686-698.

Starreveld, P. A., \& La Heij, W. (1996). Time-course analysis of semantic and orthographic context effects in picture-naming. Journal of Experimental Psychology: Learning, Memory, and Cognition, 22, 896-918.

Stemberger, J. P. (1985). An interactive activation model of language production. In A. W. Ellis (Vol. Eds.), Progress in the psychology of language, (Vol. 1). Hove, UK: Lawrence Erlbaum Associates Ltd.

Vigliocco, G., Lauer, M., Damian, M. F., \& Levelt, W. J. M. (2002a). Semantic and syntactic forces in noun phrase production. Journal of Experimental Psychology: Learning, Memory, and Cognition, 28, 46-58.

Vigliocco, G., Vinson, D. P., Damian, M. F., \& Levelt, W. J. M. (2002b). Semantic distance effects on object and action naming. Cognition, 85, B61-B69.

Vitkovitch, M., \& Tyrrell, L. (1999). The effects of distractor words on naming pictures at the subordinate level. Quarterly Journal of Experimental Psychology, 52 A, 905-926. 